\title{
CHOQUES CAMBIAIS, POLÍTICA MONETÁRIA E EQUILÍBRIO EXTERNO DA ECONOMIA BRASILEIRA EM UM AMBIENTE DE HYSTERESIS
}

Vladimir Kühl Teles ${ }^{\S}$

\begin{abstract}
RESUMO
O conceito de hysteresis sugere que um choque sobre a taxa de câmbio de um país desloca o equilíbrio do setor externo. Neste contexto, a experiência das flutuações do caso brasileiro a partir do Plano Real é examinada à luz dessa teoria, obtendo-se três conclusões centrais: 1) uma política monetária de juros altos diminui a magnitude do impacto de uma desvalorização cambial sobre as exportações, o que implica um deslocamento do equilíbrio externo; 2) uma elevação da taxa de juros aumenta a defasagem esperada das exportações com relação a choques cambiais, ou seja, aumenta a defasagem da curva J para a economia brasileira; 3) a mudança do regime cambial de câmbio fixo para flexível em 1999 alterou os parâmetros do processo estocástico que rege as flutuações cambiais, de forma a afetar os parâmetros de decisão de entrada no mercado externo das firmas domésticas e de modo a tornar necessária uma desvalorização cambial maior para a firma doméstica decidir exportar.
\end{abstract}

Palavras-chave: hysteresis, curva J, desequilíbrio externo.

\begin{abstract}
The concept of hysteresis suggests that a shock on exchange rate change the foreign equilibrium of the economy. In that context, the experience of the fluctuations of the Brazilian case from the Real Plan is examined, obtaining three central conclusions: 1) A high interest rate monetary policy diminishes the magnitude of the impact of an exchange depreciation about the exports, what imply a shift of the external equilibrium; 2) An elevation of the interest rate increase the lag of the J curve for the Brazilian economy; 3) The change of the exchange rate regime from fixed to flexible in 1999, altered the parameters of the stochastic process that governs the exchange fluctuations, affecting the entrance decision parameters in the external market of the domestic firms, being necessary a bigger exchange depreciation to the domestic firm decide to export.
\end{abstract}

Key words: hysteresis, J curve, foreign imbalance.

JEL classification: F41, F17, E52. 


\section{INTRODUÇão}

Este artigo busca explorar a relação entre dois conceitos expoentes sobre os efeitos de variações na taxa de câmbio sobre o equilíbrio externo: ${ }^{1}$ a curva J e hysteresis. Seguindo uma tradição de Dixit (1994), tal análise é realizada para a economia brasileira com a finalidade de se caracterizar tais efeitos sobre o equilíbrio externo brasileiro. Assim, o presente artigo tem como foco central da análise dois fatores que são característicos da economia brasileira no período: a mudança de regime cambial e a política monetária de juros altos.

$\mathrm{O}$ efeito denominado hysteresis tem sua origem na física, e tem sido constantemente aplicado na economia com a finalidade de se explicar mudanças no nível de equilíbrio externo causadas por choques na taxa de câmbio real da economia. Tal conceito, explorado inicialmente em macroeconomia aberta por Baldwin (1988, 1989, 1990), Baldwin e Krugman (1989), Dixit (1989, 1992) e Dixit e Pindyck (1994), considera a existência de custos de entrada em novos mercados que não podem ser recuperados posteriormente, ou, em outras palavras, sunk costs no comércio internacional.

Um efeito imediato de tais custos é que, uma vez que a economia sofre um choque na taxa de câmbio, como, por exemplo, uma sobrevalorização cambial, as firmas que decidem entrar no mercado doméstico têm de incorrer em sunk costs. Desta forma, mesmo que a taxa de câmbio retorne ao nível inicial, as firmas externas continuam exportando para a economia doméstica uma vez que já tiveram de arcar com os custos de entrada, o que causa um impacto de longo prazo sobre o equilíbrio externo da economia. ${ }^{2}$

A curva J, por sua vez, surge de uma constatação empírica de que uma desvalorização cambial causa inicialmente uma queda no saldo de transações correntes, seguida de um crescimento em tal saldo mais que proporcionalmente. A explicação teórica de tal efeito tem surgido a partir de diversas vertentes. Krueger (1983) argumenta, por exemplo, que tal fenômeno emana do fato de que no momento em que ocorre a mudança na taxa de câmbio os bens já estão sendo comercializados sob contratos que não podem ser quebrados, e que a finalização de tais transações domina as flutuações comerciais no curto prazo. Assim, embora uma depreciação tenda a deteriorar o saldo das transações correntes no curtíssimo prazo, ela tende a elevar tal saldo no médio prazo. Ao mesmo tempo, Bacchetta e Gerlach (1994) constroem um modelo teórico que é construído de forma a fazer com que a curva J surja quando os bens importados são duráveis e os preços de tais bens são rígidos.

Magee (1973) atribui o fenômeno descrito pela curva J ao fato de que o rápido crescimento da atividade doméstica relativo à atividade externa pode sobrepor qualquer efeito favorável que a desvalorização possa causar. Ele caracterizou tal fenômeno como consistindo de um período sob o qual os contratos já estão em curso sobre o câmbio determinado ex-ante, de forma a dominar o curto prazo.

Indo em um caminho contrário à explicação de rigidez nominal dada pelos autores supracitados, Backus et al. (1994) fornece uma interpretação da curva J a partir de um modelo de ciclos reais com duas economias em que as variações nos termos de troca dessas economias são causadas por choques assimétricos de produtividade.

Nesse modelo, um choque de produtividade positivo causa, num primeiro momento, um aumento de importação de bens de investimento, o que impede uma desvalorização imediata do

1 Entende-se, no presente artigo, por equilíbrio externo a evolução do saldo das transações correntes.

2 Um detalhamento sobre o efeito hysteresis sobre o equilíbrio externo pode ser encontrado em Göcke (2001, 2002). 
câmbio e o aumento das exportações, mas, em um segundo instante, quando o "boom" de importações é dissipado, a situação se reverte, configurando um comportamento semelhante ao da curva J.

Ao mesmo tempo, Junz e Rhomberg (1973) argumentam que a expansão das exportações e o retardamento das importações ocorrem apenas após um número substancial de defasagens. Eles identificaram a existência de uma defasagem de cinco anos atribuída à curva J. Segundo o referido estudo, tais defasagens estão relacionadas com diversos aspectos que seguem o choque no câmbio. Haveria defasagens no reconhecimento da nova situação, na decisão de se alterar variáveis reais, no tempo de entrega das mercadorias exportadas, na substituição tecnológica e de material e na produção.

Entretanto, a extensão das defasagens relacionadas com a curva J é motivo de discordância na literatura, uma vez que enquanto Junz e Rhomberg (1973) contabilizam tais defasagens em cinco anos, Krugman e Obstfeld (1991, p. 451) descrevem a relação inicial decrescente da curva J como limitada a menos de um ano, Bahmani-Oskooee (1985) dizem que as defasagens variam entre dois e cinco trimestres entre países, Noland (1989) verifica que estas ocorrem em um período de dois anos no ramo negativo da curva J e mais dois anos até alcançar o novo equilíbrio, e Meade (1988) demonstra que tal período pode ser maior, o que dificilmente poderia ser explicado pela curva J.

Assim, fica evidente que uma teoria bem alicerçada deve poder explicar os fatores que determinam o tamanho da defasagem relacionada à curva J. Tal explicação pode advir do comportamento inercial previsto pelo efeito hysteresis, de modo que uma conciliação da curva J com o efeito hysteresis pode fornecer um poder explicativo superior à teoria.

Nesse contexto, o presente estudo visa observar a extensão da curva J para a economia brasileira, buscando uma relação conciliatória entre a curva J e hysteresis. Justamente pela necessidade de se obter tal harmonia entre as duas teorias é que se optou pela utilização do modelo de Dixit (1994) como padrão para a presente análise, uma vez que este é o primeiro modelo a buscar tal conciliação, além de ser um modelo adequado para tal estudo. Naturalmente, a estilização do equilíbrio externo brasileiro via o modelo de Dixit (1994) torna-se capaz de fornecer uma ferramenta útil para se entender os movimentos externos, bem como para se prever direções e a duração dos efeitos de política econômica para a economia brasileira.

Assim, o artigo está organizado da seguinte forma: As duas seções a seguir se destinam a fazer uma breve recapitulação do modelo de Dixit (1989, 1994), que servirá de base para a análise. A seção quatro analisa a economia brasileira com base em tal modelo, procurando verificar como a política monetária e a mudança de regime cambial afetaram a decisão das firmas brasileiras de exportar. A quinta seção tem como foco a determinação da defasagem de um choque cambial sobre as exportações, caracterizando a curva J para a economia brasileira. A última seção destina-se aos comentários finais.

\section{FUndAMENTAÇÃO TEÓRICA}

O modelo utilizado aqui é o modelo de Dixit (1989, 1994), cuja descrição é feita no presente tópico. Considere uma firma brasileira que deseja entrar no mercado externo a um custo K. Tendo entrado, esta firma pode ofertar seu produto a um custo de $\mathrm{C}$ reais, recebendo um retorno de $\mathrm{R}$ dólares. A taxa de câmbio é dada por E reais por dólar. Logo, o fluxo de lucro em reais é dado por (ER-C), enquanto que a firma depara-se com uma taxa real interna de juros r. A taxa de câmbio real segue um movimento browniano geométrico, que é uma formulação contínua de caminho aleatório, dado por, 


$$
\frac{d E}{E}=\mu d t+\sigma d z
$$

onde $d z$ é o incremento de um processo padrão de Wiener, não autocorrelacionado, satisfazendo $E(d z)=0$ e $E\left(d z^{2}\right)=$ dt. O valor presente esperado de lucro se a firma permanecer ativa no mercado externo para sempre será de $E R /(r-\mu)-C / r$. Logo, o valor de uma firma ativa, incluindo o valor de sua opção de sair, toma a forma de,

$$
V_{A}(E)=B E^{\beta}+E R /(r-\mu)-C / r
$$

Quando inativa, a firma tem a opção de entrar no novo mercado, com o valor

$$
V_{I}(E)=A E^{-\alpha}
$$

Nessas expressões, $A$ e $B$ são constantes a serem determinadas, e $-\alpha$ e $\beta$ são raízes da equação quadrática,

$$
q(x) \equiv \frac{1}{2} \sigma^{2} x(x-1)+\mu x-r=0
$$

sob condições sobre os parâmetros $r$ e $\mu$ que garantam o ajustamento dos valores, nos termos em que $-\alpha<0$ e $\beta>1$.

A decisão ótima da firma será estabelecida a partir de dois valores limites da taxa de câmbio, $\mathrm{E}_{\mathrm{H}}$ e $\mathrm{E}_{\mathrm{L}}$, com $\mathrm{E}_{\mathrm{H}}>\mathrm{E}_{\mathrm{L}}$. Uma firma que não entrou no mercado externo decidirá pela entrada em tal mercado quando a taxa de câmbio alcançar o valor $E_{H}$, e uma firma ativa no mercado externo decide sair de tal mercado quando a taxa de câmbio cair abaixo do valor $E_{L}$, enquanto que para o intervalo entre as duas taxas limite as firmas de ambos os tipos permanecem com as suas decisões iniciais. tching,

Os valores limite $E_{H}$ e $E_{L}$, e as constantes $A$ e $B$, são definidas pelas condições de value-ma-

$$
\begin{aligned}
& V_{I}\left(E_{H}\right)=V_{A}\left(E_{H}\right)-K \\
& V_{I}\left(E_{L}\right)=V_{A}\left(E_{L}\right)
\end{aligned}
$$

e pelas condições de smooth pasting,

$$
\begin{aligned}
& V_{I}{ }^{\prime}\left(E_{H}\right)=V_{A}{ }^{\prime}\left(E_{H}\right) \\
& V_{I}{ }^{\prime}\left(E_{L}\right)=V_{A}{ }^{\prime}\left(E_{L}\right)
\end{aligned}
$$

Dessa forma, o modelo descrito é capaz de alcançar alguns resultados analíticos importantes como a relação entre hysteresis e a incerteza relacionada com o mercado externo, onde um aumento da incerteza cambial implica um aumento do efeito hysteresis. Entretanto, a maior preocupação do presente estudo é utilizar o modelo descrito para realizar exercícios de simulação numérica para a economia brasileira de forma a obtermos uma idéia melhor das propriedades da solução relacionada, bem como, e principalmente, fornecer instrumentos para a análise do equilíbrio externo do Brasil. 


\section{A DETERMinaÇão dos PARÂMETROS}

A fim de se usar o modelo de Dixit (1989) para observar o comportamento do equilíbrio externo diante de choques, o modelo descrito na seção anterior precisa ser parametrizado. Com esta finalidade, torna-se necessário determinar as equações chave para a determinação dos parâmetros. Neste sentido, a partir da equação (4) é possível determinar os valores de $\alpha$ e $\beta$, a saber,

$$
\begin{aligned}
& \beta=\frac{(1-m)+\left[(1-m)^{2}+4 \Omega\right]^{1 / 2}}{2} \\
& -\alpha=\frac{(1-m)-\left[(1-m)^{2}+4 \Omega\right]^{1 / 2}}{2}
\end{aligned}
$$

onde $m=2 \mu / \sigma^{2}$ e $\Omega=2 r / \sigma^{2}$.

Ao mesmo tempo, podemos escrever as condições (5) a (8) em termos de formas funcionais ao usar (2) e (3), e então resolver os dois sistemas subseqüentes, alcançando os valores de $E_{H}$ e $E_{L}$, dados por,

$$
\begin{aligned}
& E_{H}=\frac{r-\mu}{r} \frac{\beta}{\beta-1}(C+r K) \\
& E_{L}=\frac{r-\mu}{r} \frac{\alpha}{\alpha+1} C
\end{aligned}
$$

Dessa forma, o modelo alcança as formas funcionais necessárias para a análise numérica do caso brasileiro.

\section{O CASO BRASILEIRO}

Vamos considerar as flutuações da taxa de câmbio brasileira a partir da implementação do Plano Real em julho de 1994. Neste sentido, uma vez que ocorreu a adoção do regime de câmbio fixo como parte intrínseca do Plano Real até janeiro de 1999, quando se migrou para o regime de câmbio flutuante, as flutuações dissipam-se no período julho 1994-janeiro 1999. Dessa forma, o período pode ser dividido em dois subperíodos, cuja quebra ocorre em janeiro de 1999. 
Figura 1 - Taxa de câmbio real

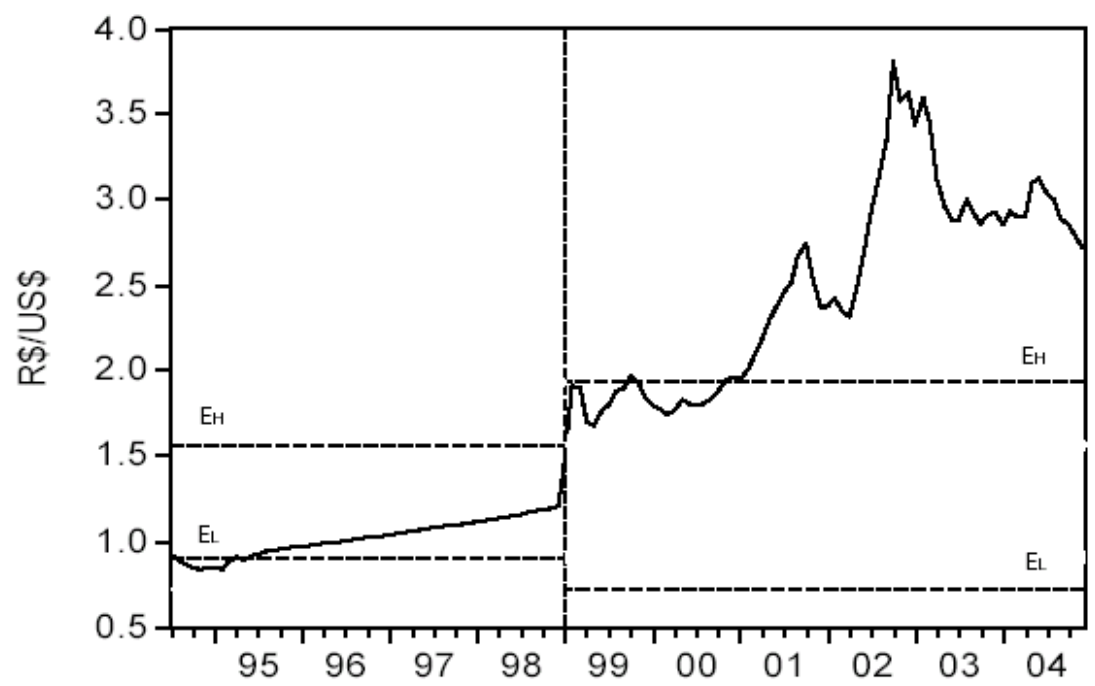

meses

Assim, usando dados mensais de julho de 1994 e dezembro de 2004 para a taxa de câmbio real cuja fonte remete-se ao IPEADATA, podemos observar as flutuações da taxa de câmbio da economia na Figura 1. Com base em tais dados foram estimados os parâmetros da equação (1) utilizando-se um modelo de séries temporais univariado para todo o período, bem como para os dois subperíodos, obtendo-se os resultados apresentados na Tabela 1.

O passo seguinte consiste em calcular os valores limites da taxa de câmbio para a entrada ou saída das empresas nacionais no mercado externo. Neste sentido, o parâmetro de custos de entrada no mercado internacional segue os padrões da literatura (e.g. Dixit, 1989) K = 4, bem como o parâmetro de custos de produção que são considerados como constituindo $95 \%$ do custo total, ou seja, $\mathrm{C}=0.95$.

Tabela 1 - Parâmetros estimados (eq. (1))

\begin{tabular}{lcc}
\hline período & $\mu$ & $\sigma$ \\
\hline $1994.07-2004.12$ & 0.0075 & 0.1022 \\
$1994.07-1998.12$ & 0.0054 & 0.0108 \\
$1999.01-2004.12$ & 0.0077 & 0.1347 \\
\hline
\end{tabular}

Ao mesmo tempo, dada a elevada variabilidade da taxa de juros no período em questão, utilizou-se um intervalo entre $5 \%$ a $25 \%$ para a taxa de juros brasileira. Assim, torna-se também possível analisar os efeitos da política de juros elevado no período sobre a decisão da firma doméstica em entrar no mercado externo, mesmo não havendo variação cambial. Os resultados obtidos para os valores limites da taxa de câmbio para os períodos delimitados são apresentados nas Tabelas 2 a 4 . Naturalmente, tais resultados estão sujeitos a críticas originadas, por exemplo, da existência de cus- 
tos de entrada, no mercado internacional, distintos para cada setor da economia, ou pela variação temporal da taxa de câmbio limite $E_{H}$.

Tabela 2 - Valores de $\mathrm{E}_{\mathrm{H}}$ e $\mathrm{E}_{\mathrm{L}}(\mathbf{1 9 9 4 . 0 7 - 2 0 0 4 . 1 2 )}$

\begin{tabular}{rcc}
\hline juros & $\mathrm{E}_{\mathrm{H}}$ & $\mathrm{E}_{\mathrm{L}}$ \\
\hline $5 \%$ & 1.4965 & 0.6210 \\
$10 \%$ & 1.6536 & 0.7221 \\
$15 \%$ & 1.8277 & 0.7656 \\
$20 \%$ & 2.0230 & 0.7911 \\
$25 \%$ & 2.2231 & 0.8084 \\
\hline
\end{tabular}

Tabela 3 - Valores de $\mathrm{E}_{\mathrm{H}}$ e $\mathrm{E}_{\mathrm{L}}(\mathbf{1 9 9 4 . 0 7 - 1 9 9 8 . 1 2 )}$

\begin{tabular}{rcc}
\hline juros & $E_{H}$ & $E_{L}$ \\
\hline $5 \%$ & 1.1616 & 0.8391 \\
$10 \%$ & 1.3626 & 0.8904 \\
$15 \%$ & 1.5637 & 0.9078 \\
$20 \%$ & 1.7647 & 0.9167 \\
$25 \%$ & 1.9656 & 0.9221 \\
\hline
\end{tabular}

Tabela 4 - Valores de $\mathrm{E}_{\mathrm{H}}$ e $\mathrm{E}_{\mathrm{L}}(\mathbf{1 9 9 9 . 0 1 - 2 0 0 4 . 1 2 )}$

\begin{tabular}{ccc}
\hline juros & $\mathrm{E}_{\mathrm{H}}$ & $\mathrm{E}_{\mathrm{L}}$ \\
\hline $5 \%$ & 1.6555 & 0.5581 \\
$10 \%$ & 1.7657 & 0.6703 \\
$15 \%$ & 1.9381 & 0.7207 \\
$20 \%$ & 2.1286 & 0.7509 \\
$25 \%$ & 2.3267 & 0.7716 \\
\hline
\end{tabular}

Os resultados das Tabelas 2 a 4 permitem extrair duas conclusões de extrema importância para a prática de política econômica para o Brasil. A primeira delas diz respeito à relação entre taxa de juros e o limite superior $E_{H}$. Neste caso, quanto maior a taxa de juros, maior deve ser a taxa de câmbio para que os agentes decidam exportar. Isto se deve ao fato de a taxa de juros elevar os custos das empresas e, portanto, é preciso um preço mais atrativo do que quando a taxa de juros está em baixos níveis. Fica assim evidente que um aumento da taxa de juros implica um forte desestímulo a exportar, uma vez que a taxa de câmbio limite, $E_{H}$, sob a qual a firma decide exportar quando esta é ultrapassada, aumenta significativa dados os aumentos na taxa de juros, mesmo que não ocorram mudanças na taxa de câmbio de equilíbrio. Portanto, uma taxa de câmbio sobrevalorizada não é o único fator a diminuir as exportações, mas também a taxa juros elevada. Em outras palavras, a des- 
valorização cambial tem seus efeitos reduzidos sobre o crescimento da exportação quando a economia encontra-se sob juros altos.

A segunda conclusão providencial refere-se à transição do regime de câmbio fixo para o regime de câmbio flutuante realizada em 1999. No que diz respeito a este aspecto, uma comparação das Tabelas 3 e 4 fornece as condições para se afirmar que a mudança no regime cambial, ao afetar os parâmetros do processo estocástico ao qual a taxa de câmbio está sujeita, determina os parâmetros de decisão de entrada no mercado externo das firmas. Ou seja, a desvalorização cambial necessária para incentivar as exportações a partir do regime de câmbio flexível é muito maior que a necessária sob o regime de câmbio fixo. Uma razão para tal efeito é a alteração drástica na variância da taxa de câmbio dos dois períodos. Neste caso, a mudança do regime cambial de fixo para flexível elevou grandemente a variabilidade da taxa de câmbio e, como conseqüência, elevou o risco da entrada no mercado externo, de forma que, nesse ambiente mais volátil, o investidor só decidirá pela entrada em um novo mercado se a taxa de câmbio for capaz de compensar o risco agora envolvido.

Esta conclusão deixa claro que a taxa de câmbio que equilibra o mercado doméstico com relação ao mercado externo, ou seja, que equilibra o saldo de Transações Correntes e o Balanço de Pagamentos, em um regime de câmbio fixo é completamente distinto da taxa necessária em um regime de câmbio flexível. Uma receita importante de política econômica para a análise que segue deste quadro é que se deve evitar um comportamento excessivamente volátil da taxa de câmbio, estabilizando-a o máximo possível, de modo a diminuir os custos necessários (em forma de desvalorização cambial) para se equilibrar o comércio com o setor externo.

Entretanto, deve-se lembrar que se a baixa volatilidade cambial diminui o limite de decisão de uma firma nacional de exportar, ela incentiva, por outro lado, o comportamento exportador das firmas estrangeiras para a economia doméstica, de modo que uma política que adota um regime de câmbio fixo sobrevalorizado, como foi o caso do Brasil entre 1994 e 1999, provoca um efeito crowding-in sobre as importações, uma vez que as importações crescerão não apenas pela valorização do câmbio, como também pela sua baixa volatilidade. Tal resultado gera um efeito claramente desalentador sobre o saldo de transações correntes, como ficou demonstrado pelos saldos observados no período (ver Silva e Andrade, 2004).

\section{A CURVA J PARA O BRASIL}

A Figura 1 mostra a realização do processo estocástico da taxa de câmbio. Entretanto, quando a taxa de câmbio mudou para o regime flexível em 1999, tal realização não era conhecida. Naquele momento, a pergunta apropriada a ser levantada seria: quanto tempo será necessário esperar até que a mudança de regime cambial tenha efeito sobre as transações correntes?

A natureza dessa questão tem sido abordada exaustivamente pela literatura referente à curva J, onde se demonstra que um choque cambial afeta o comércio com o setor externo com uma certa defasagem. Entre as possíveis explicações para tal fato encontram-se a existência da rigidez de contratos ou mesmo de uma certa rigidez real inerente ao processo produtivo. Entretanto, a presente abordagem será baseada no modelo de Dixit (1994), segundo o qual a defasagem captada na curva J é um resultado do efeito hysteresis.

A esse respeito, como demonstrado na seção anterior, uma mudança no regime cambial tende a afetar as decisões de exportar ao alterar a taxa de câmbio limite $E_{H}$, a qual as firmas nacionais observam para entrar no mercado externo. Neste sentido, a taxa de câmbio só atingirá tal limite após alguns meses, de forma a se configurar um comportamento semelhante ao predito pela curva J. As- 
sim sendo, ao aplicar o modelo de Dixit devemos considerar o logaritmo da taxa de câmbio real. Definindo-se $X=\ln E$, temos, pelo Lemma de Ito, que $X$ segue o movimento browniano dado por,

$$
d X=v d t+\sigma d z
$$

supondo que $X$ se inicie no valor $X_{0}$. Assim, o tempo esperado para este alcançar outro nível $X_{1}$ é dado por $\left(X_{1}-X_{0}\right) / v$, se $v$ e $\left(X_{1}-X_{0}\right)$ tiverem o mesmo sinal, e infinito, no caso contrário. ${ }^{3}$

Dessa forma, é possível obter que, para o período pós 1999, $v=0.002818, X_{0}=\ln 1.2046=$ 0.1861 , e $X_{1}=\ln E_{H}$, que varia de acordo com a taxa de juros escolhida. Tais valores nos levam à obtenção dos resultados constantes da Tabela 5 .

\section{Tabela 5 - Defasagem esperada (Modelo Simplificado)}

\begin{tabular}{rl}
\hline juros & Defasagem \\
\hline $5 \%$ & 9.40 anos \\
$10 \%$ & 11.30 anos \\
$15 \%$ & 14.06 anos \\
$20 \%$ & 16.83 anos \\
$25 \%$ & 19.46 anos \\
\hline
\end{tabular}

Tais resultados são extremamente elevados se comparados com os apresentados usualmente pela literatura concernente à curva J. Ademais, existe uma razão teórica que justifica a sobreestimação resultante da fórmula aplicada. Tal explicação advém da possibilidade de que, mesmo que a taxa de câmbio tenha uma tendência crescente, ela poderia, dada a sua aleatoriedade, passear por valores mais baixos do que se poderia esperar. Tal fato não seria verificado caso considerássemos que os custos de entrada no mercado externo são distintos entre setores, e que pequenas variações para cima na taxa de câmbio seriam capazes de elevar as exportações de algumas empresas, provocando novamente um impacto sobre o câmbio, de forma que é de se esperar, como destacado pelo modelo de equilíbrio geral de Dumas (1992), que a taxa de câmbio tenha um comportamento mais contínuo ao longo de sua trajetória.

Para se resolver tal problema, basta incorporar ao modelo um limite inferior para a taxa de câmbio. Neste sentido, suponhamos que $X$ se inicia em $X_{0}$. Estamos interessados em observar quanto tempo será necessário para se alcançar a taxa cambial relativa a $X_{1}$, quando a taxa cambial está sujeita a uma barreira inferior $X_{2}$, ou seja, quanto tempo a taxa cambial limite $E_{H}$ será alcançada quando $X$ está inclusa em um intervalo $\left[X_{2}, X_{1}\right]$, onde $X_{1}>X_{2}$.

Tal problema é resolvido usando-se um algoritmo de programação dinâmica, onde, partindose de $X$ durante períodos $d t$, se alcançará a taxa esperada em $\mathrm{T}(X+d X)$. Assim, torna-se necessário obter a esperança deste resultado a partir da distribuição aleatória de $d X$. Logo,

$$
T(X)=d t+E[T(X+d X)]
$$

onde $T(X)$ é o tempo esperado para se alcançar $X_{1}$.

3 A prova desta proposição encontra-se em Dixit (1993, eq. 10). 
Expandindo o lado direito da equação por meio do Lemma de Ito, e simplificando, alcançamos a equação diferencial a seguir,

$$
\frac{1}{2} \sigma^{2} T^{\prime \prime}(X)+v T^{\prime}(X)+1=0
$$

Que terá como solução geral

$$
T(X)=A+B e^{-\theta X}-X / v
$$

onde $\theta=2 v / \sigma^{2}$, e $A, B$ são constantes a serem determinadas pela utilização das condições nos pontos finais. Seguindo Dixit (1993) nós sabemos que uma destas é $T(X 1)=0$, enquanto que a condição de smooth pasting na restrição é $T^{\prime}(X 2)=0$. Logo, temos que,

$$
T(X)=\frac{\left(X_{1}-X\right)}{v}+\frac{e^{\theta X_{2}}\left[e^{-\theta X_{1}}-e^{-\theta X}\right]}{\theta v}
$$

Dessa forma, levando-se em conta que a taxa de câmbio estava extremamente sobrevalorizada antes da flexibilização em 1999, podemos considerar $X=X_{2}$, ou seja, que a taxa de câmbio inicial estava em seu limite inferior. Assim, calculando o tempo esperado a partir de (17) foram obtidos os valores dispostos na Tabela 6 .

Tabela 6 - Defasagem esperada (Modelo Restrito)

\begin{tabular}{cc}
\hline juros & Defasagem \\
\hline $5 \%$ & 4 meses \\
$10 \%$ & 6 meses \\
$15 \%$ & 10 meses \\
$20 \%$ & 14 meses \\
$25 \%$ & 19 meses \\
\hline
\end{tabular}

Os resultados apresentados na Tabela 6 fornecem um quadro bem mais realístico sobre o comportamento da taxa de câmbio na economia brasileira, o que demonstra que a imposição teórica de uma restrição inferior ao processo estocástico ao qual segue a taxa de câmbio implica uma diferença substancial na qualidade dos resultados. Tais resultados não apenas encontram-se mais próximos dos apresentados normalmente pela literatura no que concerne à curva J, como também refletem, com maior acurácia, os movimentos da taxa de câmbio na economia brasileira. Para verificar isto, basta observar que a defasagem esperada quando a taxa de juros foi fixada em 15\% (que era uma taxa de juros próxima da praticada no período) foi de 10 meses, e ao analisar os movimentos da taxa de câmbio observados na economia brasileira constatamos que a taxa de câmbio da economia ultrapassou o valor de $E_{H}$, pela primeira vez, exatamente 10 meses após a desvalorização cambial, ao atingir o valor de 1.9687 , enquanto que o $E_{H}$ obtido foi de 1.9351 . Neste sentido, a Figura 1 mostra o limite $E_{H}$ antes e após a mudança de regime cambial, tornando possível a visualização da defasagem da decisão de exportar com relação à desvalorização cambial.

Uma conclusão que pode ser obtida a partir dos resultados apresentados na Tabela 6 é que a defasagem das exportações, dada uma desvalorização cambial, aumenta para valores maiores da 
taxa de juros. Embora não seja surpreendente tal resultado, dados os resultados apresentados na seção anterior, ele é de relevância tanto para a teoria, uma vez que demonstra que a defasagem relacionada à curva $\mathrm{J}$ depende da taxa de juros da economia, quanto para a prática de política econômica, uma vez que deixa claro que uma taxa de juros elevada causa efeitos negativos magnificados sobre a taxa de câmbio e sobre o equilíbrio externo.

\section{CONCLUSÕES}

O presente artigo teve como objetivo analisar o equilíbrio externo brasileiro em um ambiente de hysteresis a partir da metodologia empregada por Dixit (1989, 1994). Neste sentido, buscou-se dar maior atenção às características intrínsecas da economia brasileira no período, a saber: a mudança de regime cambial e a política de juros altos e como estas afetaram o equilíbrio externo da economia. Logo, as principais conclusões do presente estudo referem-se a tais fatores, e podem ser discriminadas em três conclusões globais.

A primeira conclusão refere-se à relação entre política monetária e a decisão de uma firma doméstica em entrar no mercado externo. A esse respeito, ficou demonstrado que uma política de juros altos afeta negativamente a decisão de exportar. Como exemplo ilustrativo, considerando o período pós-1999, a uma taxa de juros de 5\% uma firma doméstica decidiria entrar no mercado externo se a taxa de câmbio da economia ultrapassasse a marca de 1.65, enquanto que a uma taxa de juros de $25 \%$ a firma só decidiria entrar no mercado externo se o câmbio ultrapassasse a taxa de 2.32. Em outras palavras, uma desvalorização tem seus efeitos reduzidos sobre as exportações quando a taxa de juros for elevada.

A segunda conclusão relaciona-se com a observação de que a mudança de regime cambial de câmbio fixo para câmbio flexível em 1999 altera a taxa de câmbio limite sob a qual as firmas domésticas decidirem começar a exportar. Neste contexto, a mudança de regime cambial mudou decisivamente os parâmetros do processo estocástico ao qual a taxa de câmbio está sujeita, como a sua tendência e sua variância. Como resultado, não apenas a taxa de câmbio esperada mudou, mas também a volatilidade esperada da taxa de câmbio, elevando o risco da entrada de firmas domésticas no setor externo. Desta maneira, uma firma só decidirá pela entrada em um novo mercado se a taxa de câmbio for capaz de compensar o risco agora envolvido.

Por fim, a terceira conclusão se relaciona à defasagem descrita pela curva J. Assim, pela presente análise foi possível calcular a defasagem esperada de um choque cambial sobre as exportações das firmas. Tal defasagem está diretamente relacionada com a taxa de juros da economia, indicando que um aumento da taxa de juros tende a elevar tal defasagem, ou seja, a defasagem relacionada à curva $\mathrm{J}$ depende da política monetária adotada. Neste ponto, a acurácia do modelo foi posta à prova quando foram comparados os valores da defasagem prevista pelo modelo com a ocorrida em 1999, comprovando que embora não seja objetivo do estudo fornecer um instrumento literal de previsão, os valores determinados pelo modelo se aproximam da realidade observada.

\section{BIBLIOGRAFIA}

Bachetta, P.; Gerlach, S. Sticky import prices and J-curves. Economics Letters 44, p. 281-285, 1994.

Backus, D. et al. Dynamics of the trade balance and the terms of trade: the J-curve?. American Economic Review 84, p. 84-103, 1994. 
Bahmani-Oskooee, M. Devaluation and the J-curve: some evidence from LDCs. The Review of Economics and Statistics, v. 67, n. 3, p. 500-504, 1985.

Baldwin, A. Hysteresis in import prices: the beachhead effect. American Economic Review, 78, p. 773 785, 1988.

. Sunk-cost hystersis. NBER Working Paper 2911, 1989. p.19-34.

Hysteresis in trade. In: Franz, W. (ed.), Hystersis effects in economic models. Heidelberg, 1990,

Baldwin, A.; Krugman, P. Persistent trade effects of large exchange rate schoks. Quartely Journal of Economics, 104, p. 635-54, 1989.

Dixit, A. Entry and exit decisions under uncertainty. Journal of Political Economy, 97, p. 620-38, 1989.

. Investment and hysteresis. Journal of Economic Perspectives, 6, p. 107-32, 1992.

. The art of smooth pasting. In: Lesourne, J.; Sonnenschein, H. (eds.), Fundamental of pure and applied economics. Harwood Academic Publishers, Reading, UK, 1993. (v. 55 of monograph series).

. Hysteresis and the duration of the J-curve. Japan and the World Economy, 6, p. 105-115, 1994.

Dixit, A.; Pyndick, R. Investment under uncertainty. Princeton, NJ: Princeton University Press, 1989.

Dumas, B. Dybamic equilibrium and the exchange rate in a spatially separated world. Review of Financial Studies, 5, p. 153-180, 1992.

Göcke, M. A macroeconomic model with hysteresis in foreign trade. Metroeconomica, v. 52, n. 4, p. 44973, 2001.

Various concepts of hysteresis applied in economics. Journal of Economic Surveys, v. 16, n. 2. p. 167-188, 2002.

Junz, H.; Rhomberg, R. Price competitiveness in export trade among industrial countries. American Economic Review, 63, p. 412-418, May 1973.

Krueger, A. Exchange rate determination. Cambridge: Cambridge University Press, 1983.

Krugman, P.; Obstfeld, M. International economics. 2nd ed. NY: Harper Collins, 1991.

Magee, S. Currency contracts, pass through and devaluation. Brooking Papers on Economic Activity, 1, p. 303-25, 1973.

Meade, E. E. Exchange rates, adjustment, and the J curve. Federal Reserve Bulletin, 74, p. 633-44, 1988.

Noland, M. Japanese trade elasticities and the J-curve. The Review of Economics and Statistics, v. 71, n. 1, p. 175-179, 1989.

Silva, N.; Andrade, J. Modelo intertemporal de conta corrente: evidências para o Brasil. Anais do XXXII Encontro Nacional de Economia da ANPEC. João Pessoa-PB, 2004. 